\title{
Sequencing-Based Protein Analysis of Single Extracellular Vesicles
}

Jina Ko1,2,\#, Yongcheng Wang 2,3,4,\#, Kuanwei Sheng2 ${ }^{2}$, David A. Weitz ${ }^{2,3}$, Ralph Weissleder1,5*

1 Center for Systems Biology, Massachusetts General Hospital, 185 Cambridge St, CPZN 5206, Boston, MA 02114, USA

2 Wyss Institute for Biologically Inspired Engineering, Harvard University, Boston, MA 02115, USA

3 John A. Paulson School of Engineering and Applied Sciences and Department of Physics, Harvard University, Cambridge, MA 02138, USA

4 Department of Chemistry and Chemical Biology, Harvard University, Cambridge, MA 02138, USA

${ }^{5}$ Department of Systems Biology, Harvard Medical School, 200 Longwood Ave, Boston, MA 02115, USA

\# These authors contributed equally to this work.

${ }^{*}$ R. Weissleder, MD, PhD

Center for Systems Biology

Massachusetts General Hospital Research Institute

185 Cambridge St, CPZN 5206

Boston, MA, 02114

617-726-8226

rweissleder@mgh.harvard.edu 
Figure S1. Bead-DNA $A_{B C}$ and $A b-D N A_{B C}$ sequences. The bead-DNA $A_{B C}$ sequence consists of the sequence shown including [bc1'], [bc2'], and [bc3'] regions and there are 96, 96, and 24 barcodes respectively. The $A b-D N A_{B C}$ sequence consists of [Ab barcode] region that include 10 different sequences as shown in the table. Corresponding antibodies that were conjugated to the Ab-DNABC are included.

Bead-DNABc: GTGAGTGATGGTTGAGGATGTGTGGAGATA [bc1'] TGGTAGGTGGTAGAGA [bc2'] AGGTATGGTGATTGTG [bc3'] NNNNNNGAGAATGTGAGTGAAGATGTATGGTGA

Ab-DNABc: TAATACGACTCACTATAGGGAGATGGAGGGTGTGTAGT [Ab barcodes 1-10] TCACCATACATCTTCACTCACATTCTC

\begin{tabular}{|c|c|c|}
\hline [Ab barcode] & Sequence & Target protein / antibody \\
\hline 1 & ACCGT & EGFR \\
\hline 2 & CATACA & Human IgG1 isotype control \\
\hline 3 & CGATGA & CD9 \\
\hline 4 & GCATAT & F4/80 \\
\hline 5 & TGCGTG & Rat IgG2a isotype control \\
\hline 6 & AGGCGT & Rat IgG2b isotype control \\
\hline 7 & GAGGCA & CD11b \\
\hline 8 & ATTGAG & CD63 \\
\hline 9 & ATAAGA & CD45 \\
\hline 10 & GTGAGA & CD81 \\
\hline
\end{tabular}


Figure S2. Chip design. A design of the droplet generator used for this study is included with dimensions (droplet collection region: $140 \mu \mathrm{m} \mathrm{W}$, oil input: $60 \mu \mathrm{m} \mathrm{W}$, bead input: $40 \mu \mathrm{m} \mathrm{W}$, EV input: $55 \mu \mathrm{m} \mathrm{W}$, master mix input: $60 \mu \mathrm{m} \mathrm{W}$ ).

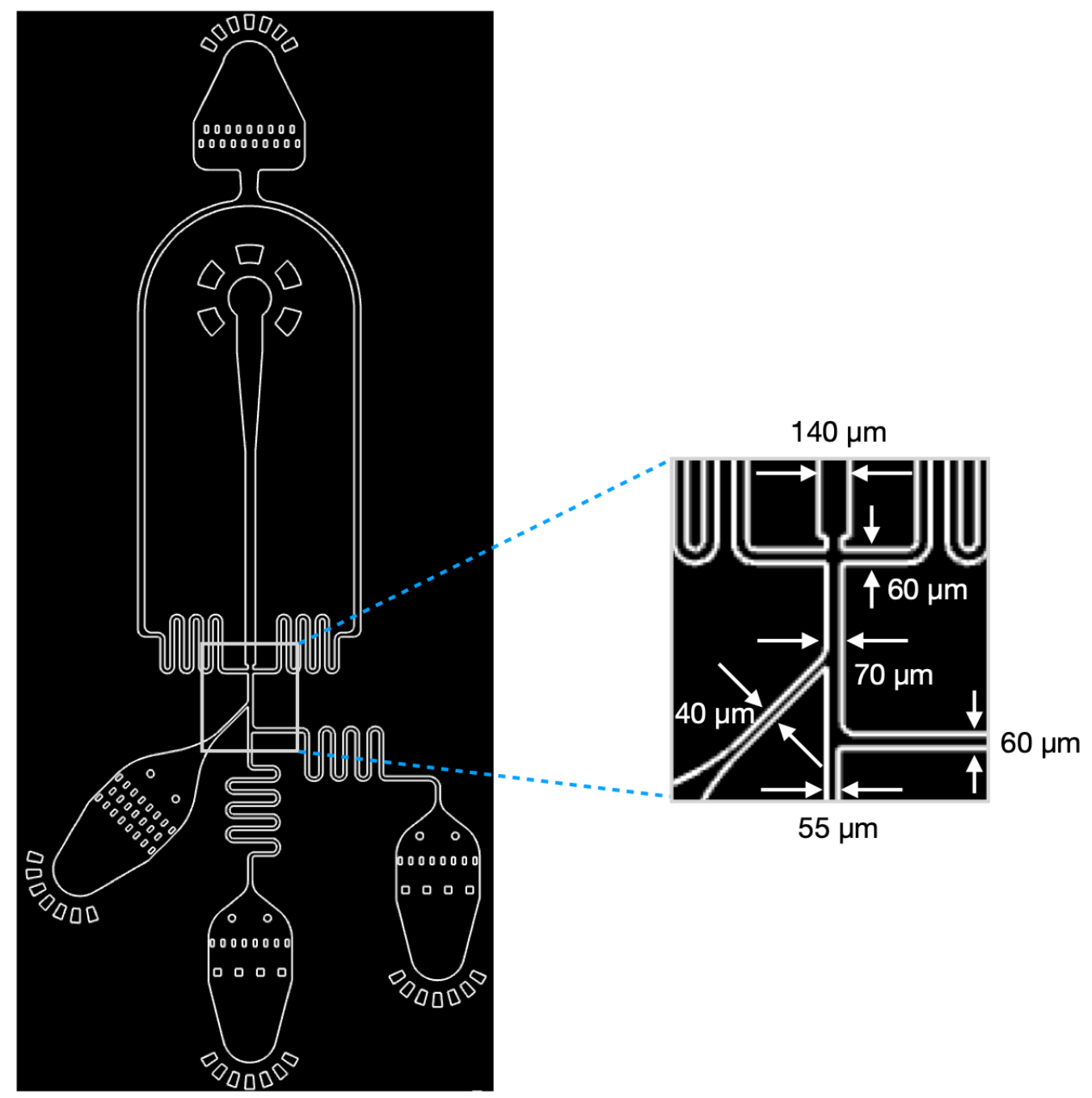


Figure S3. Control experiment antibody specificity. An anti-EGFR antibody (human IgG1) and its matching isotype control (human IgG1) were used to compare the number of reads. Histograms with individual EV ( $x$-axis) and their corresponding reads ( $y$-axis) were created for both isotype control and EGFR samples. The threshold in the isotype control data was set at the $95 \%$ confidence interval which corresponded to EV with 2 reads. Therefore, for EGFR, all EV with more than 2 reads were considered to be specific and analyzed ( $775 \mathrm{EV}$ from 3899 total EV). Isotype control was included for each experiment and its own threshold was determined and applied for future analysis.

Isotype control

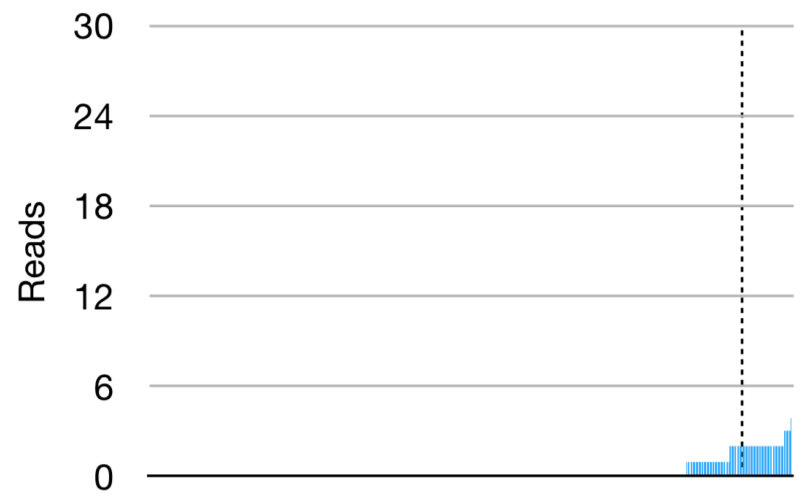

EGFR

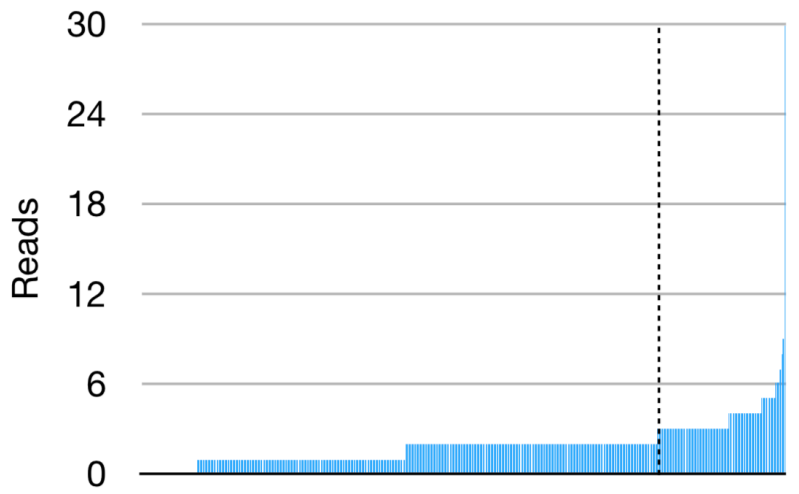

